\title{
Aproximación a la medición de la percepción de riesgo en la acuacultura de Sonora, México
}

\author{
Araiza-Sánchez, L.M. ${ }^{a}$, Valenzuela-Valenzuela A. c, Laborín-Alvarez, J.F. ${ }^{a}$, Ortega-García, J. ${ }^{\text {, }}$ \\ Borbón-Morales C.G. ${ }^{\text {** }}$ Rueda-Puente E.O. ${ }^{\text {b* }}$ \\ a Centro de Investigación en Alimentación y Desarrollo \\ b Universidad de Sonora, Departamento de Agricultura y Ganadería \\ c Universidad de Sonora, Departamento de Ingeniería Industrial \\ d Departamento de Ciencias Químico Biológicas y Agropecuarias de la Unidad Regional Norte de la Universidad de Sonora.
}

\section{Resumen}

La producción de camarón en acuacultura presenta riesgos naturales asociados; embate de epizootias, precios internacionales bajos, y una fuerte competencia internacional. Son causas relevantes que han impactado en la disminución de la rentabilidad de las unidades productoras de camarón. Este análisis aborda otro aspecto que, eventualmente afecta su desempeño. Tales son las formas en las cuales los productores de camarón, su equipo de administradores y técnicos, toman las decisiones, y cómo estos afrontan los riesgos inherentes a esta actividad. Producto de heurísticos del pensamiento, sesgos cognitivos y la racionalidad limitada de los individuos, en ámbitos de información incompleta, inadecuada o desfasada, la toma de decisiones de estos agentes económicos, está matizada con el reto de mantener la unidad económica en funcionamiento. Con el objetivo de distinguir las formas de pensamiento que afectan a los tomadores de decisiones y encontrar un instrumento que permita medir la percepción del riesgo, se realiza una revisión sistemática, que explora las vertientes teóricas y sus hallazgos sobre el afrontamiento ante el riesgo extremo. La Teoría Prospectiva de Kahneman y Tversky (1979), es el punto de partida para establecer un parámetro claro de que esos heurísticos inherentes a la racionalidad humana.

Palabras clave:Aprovechamiento, formas de pensamiento, rentabilidad, toma de decisión.

\section{An approach to the measurement of risk perception in decision making in aquaculture in Sonora, Mexico}

\begin{abstract}
Shrimp aquaculture production presents associated natural risks; onslaught of epizootics, low international prices, and strong international competition. These are relevant causes that have impacted on the decrease in the profitability of shrimp producing units. This analysis addresses another aspect that eventually affects your performance. Such are the ways in which shrimp producers, their team of managers and technicians, make decisions, and how they face the risks inherent in this activity. Product of thought heuristics, cognitive biases and the limited rationality of individuals, in areas of incomplete, inadequate or outdated information, the decision making of these economic agents is nuanced with the challenge of keeping the economic unit in operation. In order to distinguish the ways of thinking that decision makers see and find an instrument that allows to measure risk perception, a systematic review is carried out, which explores the theoretical aspects and their findings on coping with extreme risk. The Prospective Theory of Kahneman and Tversky (1979), is the starting point to establish a clear parameter that those heuristics inherent to human rationality.
\end{abstract}

Keywords: exploitation, ways of thinking, profitability, decision making.

*Autor para envío de correspondencia: Centro de Investigación en Alimentación y Desarrollo, Carretera a La Victoria km 0.6. C.P. 83304, Hermosillo, Sonora, México. Teléfono +52 (662) 289-24-00. E-mail: cborbon@ciad.mx, erueda04@santana.uson.mx

(c) 2020 Editorial UNISON — URN. Derechos reservados. 


\section{INTRODUCCION}

Se estima que, en la producción mundial, la acuicultura representó el $46 \%$ de la producción total (FAO, 2020), de los cuatro grupos de mayor valor: atunes, cefalópodos, langosta y camarones, de estos últimos, Penaeus vannamei es la especie preferida y la más utilizada para su cultivo. De ahí que la producción en acuacultura contribuye a la producción de una fuente adicional de proteína para el consumo humano, favorece la seguridad alimentaria, genera divisas, impulsa la creación de nuevas fuentes de empleo, y reduce la presión sobre los recursos naturales particularmente en áreas costeras, generando desarrollo regional. (FA0, 2005).

Al igual que otras actividades productivas, la acuacultura está sujeta a la incidencia de factores internos y externos que complican la toma de decisiones, acotan su productividad y por consiguiente su rentabilidad. En este sentido, en un ambiente de negocios en constante cambio, no es posible disponer de toda la información para tomar decisiones, que permitan una constante maximización de utilidades y un mejor posicionamiento competitivo. El presente documento, pretende caracterizar los tipos de riesgos a los que se exponen los camaronicultores. Asimismo, detectar un instrumento psicométrico que permita la medición del afrontamiento ante la percepción del riesgo de los agentes económicos involucrados en la toma de decisiones administrativas y de producción.

Lo anterior, mediante una aproximación sistemática de revisión bibliográfica de bases de datos como: Redalyc, Scielo y SCOPUS. Las secciones que se abordan son: Antecedentes, que involucra a un panorama internacional, nacional y estatal de la producción de camarón en acuacultura; los riesgos en la cadena de producción y de mercado; la descripción de la toma de decisiones como un proceso influenciado por la percepción ante el riesgo de los agentes económicos; y finalmente, los hallazgos del análisis sistemático y la descripción del instrumento psicométrico.

\section{ANTECEDENTES}

De acuerdo a Bartolini y Torres (2004), existen tres tipos de camaronicultura, en América Latina y en México. El sistema extensivo, el más utilizado, consiste en la engorda de camarones a bajas densidades en múltiples estanques de gran extensión, en los que existe poco o nulo control de las condiciones de los diferentes factores ambientales que interactúan en el medio ambiente. El segundo es el sistema semi-intensivo, tiene densidades de siembra moderados y la manipulación de los factores que intervienen en el sistema se hace de manera moderada. El tercer sistema es el intensivo, con altas densidades de siembra y los factores que intervienen son controlados intensamente.

Actualmente el más utilizado en el Noroeste de México es el primero, lo que aporta en gran medida un escenario de producción de camarón que es de alto riesgo e incertidumbre económica, debido a factores intrínsecos a la producción de organismos vivos, pero especialmente por factores externos como variables ambientales, de mercado y enfermedades (principalmente virales), entre otros.

En una perspectiva amplia, las fuentes de incertidumbre en las que incurre esta actividad, son aquellas en las cuales los tomadores de decisiones no tienen la capacidad de incidir sobre ellas, tales como los cambios de precios, la disminución de la demanda u oferta de insumos, precios de los insumos, políticas públicas. El incremento de los costos de producción y la volatilidad de los precios en los mercados finales, entre otros, así como los esquemas de administración que en ocasiones no son lo más eficiente en el manejo de capital y trabajo; entre los más relevantes.

\section{PANORAMA INTERNACIONAL, NACIONAL Y ESTATAL DE LA PRODUCCIÓN DE CAMARÓN EN ACUACULTURA.}

\section{La perspectiva de crecimiento antes de la pandemia del COVID-19.}

Para el año 2020, los pronósticos de instituciones gubernamentales y asociaciones de productores, tenían una perspectiva halagüeña para la producción de camarón. Así, la Alianza Mundial de Acuacultura, mantenía una expectativa optimista de crecimiento del $5.7 \%$ anual promedio entre 2017 y 2020. Por su parte, el Servicio Nacional de Pesca Marina de la Agencia de Administración Nacional Oceánica y Atmosférica del Departamento de Comercio de los Estados Unidos, registraba crecimiento en la actividad.

A nivel nacional, el FIRA (2020), que continuarían en crecimiento países como India, Vietnam, Indonesia, y China, donde se concentra más del $74.8 \%$ de la producción de camarón de cultivo. Por su parte, en América Latina, se esperaba un crecimiento en la producción de: Ecuador 500 mil toneladas, México 177 mil toneladas y Brasil 60 mil toneladas.

A partir de la irrupción de la pandemia del COVID-19, la producción de camarón ha mostrado una franca caída. El efecto inmediato visible de este fenómeno es la caída de precios internacional. El caso específico de Sonora, según cifras oficiales en el año 2018 ocupó el segundo lugar en 
producción generando un 26\% del producto total nacional, después del estado de Sinaloa, que aportó en el mismo periodo un $34 \%$ de la producción. Mientras que el tercer lugar lo ocupó el estado de Nayarit, con un equivalente al $4 \%$ del producto nacional (SIACON, 2019). En resumen, estos tres estados contribuyen anualmente con más del $80 \%$ de la producción de camarón de acuacultura del país (Tabla $1)$.

Tabla 1. México: Producción de camarón en acuacultura y pesca, 2018.

\begin{tabular}{lccc} 
& \multicolumn{3}{c}{ Peso vivo en Toneladas } \\
\hline Estados & Acuacultura & Pesca & Total \\
\hline Sinaloa & 78,613 & 26,408 & 105,021 \\
\hline Sonora & 58,823 & 12,323 & 71,146 \\
\hline Nayarit & 9,800 & 7,833 & 17,633 \\
\hline Colima & 4,276 & N/D & 4,276 \\
\hline Baja California Sur & 3,229 & 1,594 & 4,823 \\
\hline Otros & 3,027 & 19,219 & 22,247 \\
\hline Total & 157,769 & 67,376 & 225,146 \\
\hline
\end{tabular}

Fuente: SIACON 2019.

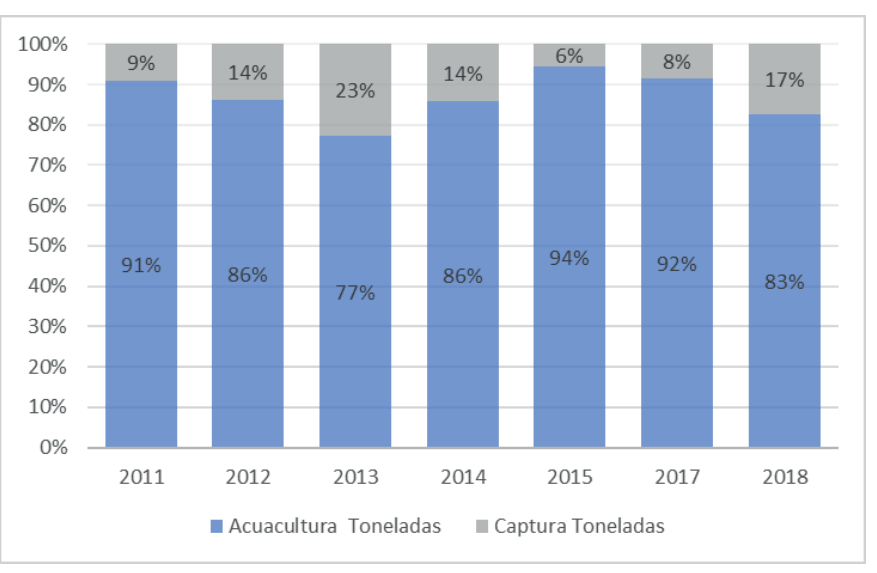

Figura 1. Sonora: Evolución del valor de producción de camarón acuacultura y captura, 2011-2018. Fuente: SIACON 2019.

En una situación antes del COVID-19, la producción total de camarón en México alcanzaría las 249.5 mil toneladas, es decir, un crecimiento anual del $5.0 \%$ del total de la producción, el $71.1 \%$ provendría de la camaronicultura. (CONAPESCA, 2017).

\section{Riesgos que asumen las Unidades de Producción Camaronícola (UPC).}

Los riesgos que tradicionalmente asume esta actividad, están relacionados con la cadena de producción y la comercialización del producto.

\section{Riesgos en la cadena productiva camaronícola}

De acuerdo a Higuera I. y Chávez M. (2013), los factores de riesgo involucrados están relacionados con: a) selección del sitio (agua y suelo de mala calidad); b) uso de alimento de mala calidad; c) introducción de patógenos que pueden ocasionar zoonosis (enfermedades de los animales que son transmitibles al hombre) obligando al uso de agentes químicos y/o tratamientos terapéuticos y d) contaminantes y/o toxinas provenientes del medio ambiente que pueden afectar la inocuidad del producto cosechado. De los anteriores aspectos, resalta el impacto de las epizootias y enfermedades, Tabla 2.

Tabla 2. Aparición de enfermedades infecciosas de camarón en México

\begin{tabular}{lc} 
Enfermedad & Año \\
\hline $\begin{array}{l}\text { Síndrome de Tauravirus (TSV) } \\
\text { Hepatopancreatitis necrotizante (NHP) }\end{array}$ & 1990 \\
\hline $\begin{array}{l}\text { Necrosis infecciosa hipodérmica y hematopoyética } \\
\text { (IHHNV) }\end{array}$ & 1998 \\
\hline $\begin{array}{l}\text { Enfermedad síndrome de las manchas blancas (WSSV) } \\
\text { Síndrome de la Muerte Temprana }\end{array}$ & 1999 \\
\hline $\begin{array}{l}\text { Enfermedad de Necrosis Hepatopancreática Aguda } \\
\text { (EMS/AHPND) }\end{array}$ & 2014 \\
\hline
\end{tabular}

Fuente: Gómez-Gil et al., 2001; en Bonett-Calzada 2017.

\section{Riesgos de Mercado: competencia internacional y bajos precios internacionales}

Según USTIC (2020), el país productor preferido por los consumidores estadounidenses había sido Tailandia, quien por más de 20 años hasta 2011 mantuvo liderazgo. Pero a partir de la denominada crisis de los antibióticos, vio disminuidas sus ventas en casi un $70 \%$ a niveles que en 2019. La situación en gran medida ha dado lugar al crecimiento y posicionamiento de India e Indonesia, ya que actualmente acaparan más del $60 \%$ las importaciones de EEUU. Ambas naciones, en 1989 abastecían menos del $10 \%$ de la demanda, mientras México cubría el $25 \%$ de la demanda. Treinta años más tarde, para 2019, la cuota mexicana cae hasta un margen apenas superior al $6 \%$.

Un hecho fehaciente es que no ha habido una recuperación significativa de los precios de exportación desde la caída de los precios en abril de 2018. (FAO, 2019). Esta tendencia 
ha continuado, ya que de acuerdo a la evolución de los precios del camarón de exportación Shrimp, No.1 de origen mexicano (Figura 3), la influencia del Covid-19 ha presentado una caída de -14\% desde noviembre 2019 a septiembre de 2020.

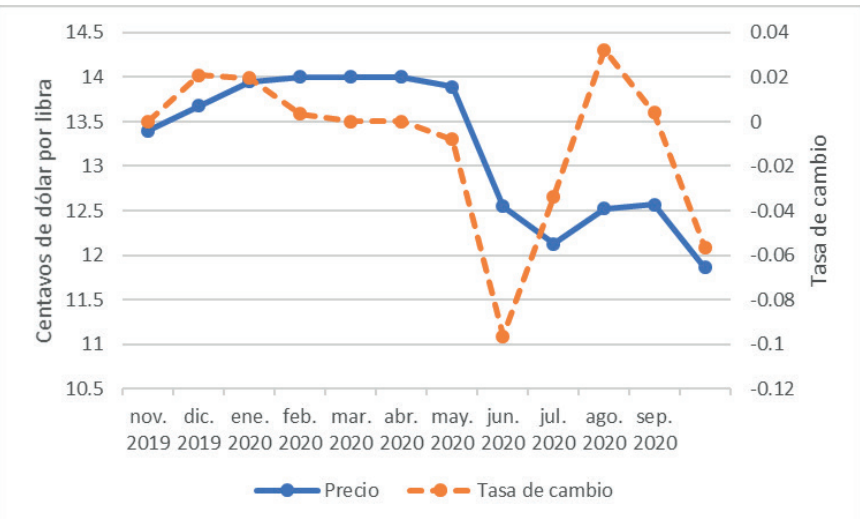

Figura 3. Evolución de los precios internacionales de camarón Shrimp, No.1 de origen mexicano (nov 2019-sep 2020). Fuente: https://www.indexmundi.com/es/preciosde-mercado/?mercancia $=$ camaron La toma de decisiones: un proceso influenciado por la
percepción ante el riesgo

La toma de decisiones en la administración de la producción camaronícola está sujeta tanto a factores internos, como a elementos externos. En cuanto a los primeros, se encuentran su estructura administrativa-técnica de proceso productivo. Respecto a los segundos, se encuentran las condiciones del ambiente de mercado o factores externos, (Tabla 3).

Tabla 3. Algunos factores que afectan la toma de decisiones

\begin{tabular}{|c|c|}
\hline Riesgos Externos & Riesgos Internos \\
\hline Competencia & Financiero \\
\hline Precios internacionales & Administrativo \\
\hline Costos de insumos & Productividad \\
\hline Epizootias & Tecnología \\
\hline
\end{tabular}

Fuente: Elaboración propia.

La toma de decisiones es el proceso para identificar y seleccionar un curso de acción para resolver un problema específico (Stoner et al, 2000). La teoría prospectiva de Kahneman y Tversky distingue dos fases en el proceso de elección: preparación y evaluación. (Tabla 4).
Tabla 4. Fases de la toma de decisiones

\begin{tabular}{|c|c|c|c|c|}
\hline \multirow[b]{2}{*}{ Preparación } & Fase 1 & \multirow[b]{2}{*}{ Combinación } & \multicolumn{2}{|c|}{ Fase 2} \\
\hline & Codificación & & Separación & Cancelación \\
\hline $\begin{array}{l}\text { Organizar y } \\
\text { reformular } \\
\text { opciones para } \\
\text { simplificar la } \\
\text { evaluación } \\
\text { y elección, } \\
\text { mediante } \\
\text { operaciones } \\
\text { que } \\
\text { transforman los } \\
\text { resultados y las } \\
\text { probabilidades } \\
\text { asociadas con } \\
\text { las alternativas } \\
\text { que se } \\
\text { presentan. }\end{array}$ & $\begin{array}{l}\text { Las ganancias } \\
\text { y pérdidas se } \\
\text { definen según } \\
\text { un punto de } \\
\text { referencia } \\
\text { neutral, la } \\
\text { codificación } \\
\text { de resultados, } \\
\text { puede verse } \\
\text { afectada por } \\
\text { la formulación } \\
\text { de las } \\
\text { alternativas } \\
\text { que se } \\
\text { ofrecen y por } \\
\text { expectativas } \\
\text { del decisor. }\end{array}$ & $\begin{array}{l}\text { Las alternativas, } \\
\text { a menudo, } \\
\text { pueden } \\
\text { simplificarse } \\
\text { combinando } \\
\text { las } \\
\text { probabilidades } \\
\text { asociadas con } \\
\text { resultados } \\
\text { idénticos. }\end{array}$ & $\begin{array}{l}\text { Algunas } \\
\text { alternativas } \\
\text { contienen } \\
\text { componentes } \\
\text { que no } \\
\text { implican } \\
\text { riesgo que } \\
\text { son separados } \\
\text { de los } \\
\text { componentes } \\
\text { con riesgo } \\
\text { en la fase de } \\
\text { preparación. }\end{array}$ & $\begin{array}{l}\text { Es la } \\
\text { eliminación } \\
\text { de } \\
\text { componentes } \\
\text { compartidos } \\
\text { por las } \\
\text { alternativas } \\
\text { que se } \\
\text { ofrecen. } \\
\text { Otro tipo de } \\
\text { cancelación } \\
\text { implica la } \\
\text { eliminación } \\
\text { de los } \\
\text { elementos } \\
\text { comunes. }\end{array}$ \\
\hline
\end{tabular}

Algunas operaciones de la fase de preparación pueden permitir o impedir la aplicación de otras.

Fuente: Elaboración propia basado en Kahneman y Tversky (1979).

\section{Heurísticos del pensamiento, sesgos cognitivos $y$ racionalidad limitada.}

De acuerdo a la Economía del Comportamiento, la heurística como característica humana de toma de decisiones se basa en aproximaciones empíricas que tienen que ver con sesgos cognitivos y racionalidad limitada (Mullainathan, 2000; Ariely 2009; Kanheman, 2012).

En este sentido, se puede inferir que los administradores toman decisiones a partir de razonamientos de tipo intuitivo. En un ambiente de negocios en constante cambio, no es posible disponer de toda la información concluyente para tomar una decisión maximizadora. En el contexto de la acción, la incertidumbre, es un sentido de duda que bloquea o retrasa tal acción (Lipshitz and Strauss, 1997). Esto obliga al administrador a recurrir a heurísticos; estrategias que ignoran información para tomar decisiones más rápido, más prudentemente y/o más precisas que con los métodos complejos (Gigerenzer, 2011).

\section{Análisis sistemático en búsqueda de la medición del afrontamiento al riesgo.}

Mediante un análisis sistemático de la bibliografía en Redalyc, Scielo y SCOPUS, se realizaron búsquedas sobre las siguientes temáticas: Toma de decisiones, heurísticos y sesgos cognitivos, racionalidad limitada, competitividad, afrontamiento ante el riesgo. Se puede apreciar que en Tabla 5,6 y 7, la cadena de producción acuícola del camarón y el sesgo, heurístico y posible afrontamiento al riesgo y una 
escala de Afrontamiento ante la percepción de un riesgo (Échelle Toulousaine de Coping), respectivamente. En la primera resaltando aquellas apreciaciones por BastidasPlaza (2006), indicando que el mercado del camarón requiere mejores procesos de producción con la finalidad de alcanzar una ventaja competitiva y que es fundamental poder diseñar un sistema de planeación y control que permita ver el comportamiento de todo el negocio en una cadena de producción acuícola del camarón (Tabla 5).
Asimismo, la heurística ha jugado un papel importante en la 'búsqueda de soluciones al riesgo, basándose como la capacidad que tiene el hombre de crear o inventar algo, con la finalidad de proporcionar estrategias que ayuden a la resolución de un problema. Los seres humanos a través de su creatividad, pensamiento divergente y en algunos casos de experiencias propias, son capaces de encontrar la solución más viable para resolver algún conflicto (Tabla 6 y 7).

Tabla 5. La cadena de producción acuícola del camarón.

\begin{tabular}{|c|c|c|c|}
\hline Autor(es) & Título/Año & Objetivo & Conclusiones \\
\hline $\begin{array}{l}\text { Héctor Eduardo } \\
\text { Bastidas Plaza } \\
(2006)\end{array}$ & $\begin{array}{l}\text { Análisis del proceso de } \\
\text { producción de camarón } \\
\text { como herramienta para la } \\
\text { toma de decisiones en los } \\
\text { agronegocios. }\end{array}$ & $\begin{array}{l}\text { Analizar el proceso productivo del cultivo } \\
\text { de camarón con un enfoque administrativo, } \\
\text { evaluando la respuesta de crecimiento en peso } \\
\text { individual (gr) y alimento suministrado ( } \mathrm{kg} / \mathrm{ha} \text {.), } \\
\text { con el fin de buscar alternativas de medición } \\
\text { de los procesos para su mejor comprensión, } \\
\text { control y toma de decisiones. }\end{array}$ & $\begin{array}{l}\text { Se considera que el mercado del camarón } \\
\text { requiere mejores procesos de producción } \\
\text { con la finalidad de alcanzar una ventaja } \\
\text { competitiva. Es fundamental poder diseñar } \\
\text { un sistema de planeación y control que } \\
\text { permita ver el comportamiento de todo el } \\
\text { negocio. }\end{array}$ \\
\hline $\begin{array}{l}\text { Ernesto A. } \\
\text { Lagarda-Leyva } \\
(2016)\end{array}$ & $\begin{array}{l}\text { Evaluación del } \\
\text { desempeño de la } \\
\text { cadena de suministro del } \\
\text { camarón blanco de agua } \\
\text { dulce. / (2016). }\end{array}$ & $\begin{array}{l}\text { Desarrollar un modelo de dinámica de } \\
\text { sistemas, para las Pymes del municipio de } \\
\text { Cajeme que permita evaluar el desempeño de } \\
\text { la cadena de suministro en frío del camarón } \\
\text { blanco y apoye en la toma de decisiones } \\
\text { empleando la metodología de dinámica de } \\
\text { sistemas y escenarios del sector económico } \\
\text { secundario. }\end{array}$ & $\begin{array}{l}\text { Implementación de las etapas aplicadas a } \\
\text { la cadena de suministro en un producto } \\
\text { regional, sobre la metodología de Forrester } \\
\text { del MIT, que incluye cuatro fases: } \\
\text { conceptualización, formulación, simulación } \\
\text { y análisis de sensibilidad. }\end{array}$ \\
\hline $\begin{array}{l}\text { Manuel Muñoz } \\
\text { Palma, Ezequiel } \\
\text { Avilés Ochoa, } \\
\text { Eva L. Miranda } \\
\text { Espinoza(2016) }\end{array}$ & $\begin{array}{l}\text { La Lógica Difusa para la } \\
\text { evaluación económica y } \\
\text { financiera de opciones } \\
\text { cambiarias: El caso de la } \\
\text { producción acuícola. / } \\
2016 .\end{array}$ & $\begin{array}{l}\text { Diseñar estrategias financieras que permitan } \\
\text { que las organizaciones acuícolas del sur de } \\
\text { Sonora puedan establecer un sistema de } \\
\text { control y evaluación del riesgo financiero, por } \\
\text { medio de instrumentos de cobertura, cuya } \\
\text { finalidad es minimizar el riesgo e incrementar } \\
\text { los índices de rentabilidad }\end{array}$ & $\begin{array}{l}\text { La lógica difusa en problemas de decisión } \\
\text { de la actividad empresarial posibilita analizar } \\
\text { una gama de alternativas al momento de } \\
\text { la evaluación financiera de proyectos que } \\
\text { presenten alto grado de incertidumbre, } \\
\text { con la finalidad de captar con precisión la } \\
\text { percepción humana. }\end{array}$ \\
\hline
\end{tabular}

b) Heurísticos, sesgos y afrontamiento al riesgo.

Tabla 6. Sesgo, heurístico y posible afrontamiento al riesgo

\begin{tabular}{|c|c|c|c|}
\hline $\begin{array}{l}\text { M. Asher Lawson, } \\
\text { Richard P. Larrick, } \\
\text { Jack B. Soll } \\
(2020)\end{array}$ & $\begin{array}{l}\text { Comparing fast thinking and slow } \\
\text { thinking: The relative benefits of } \\
\text { interventions, individual differences, } \\
\text { and inferential rules./ Judgment and } \\
\text { Decision Making, Vol. } 15 \text {, No. 5, } \\
\text { September 2020, pp. } 660-684\end{array}$ & $\begin{array}{l}\text { Examinar los beneficios de ralentizar el } \\
\text { pensamiento para la toma de decisiones } \\
\text { individuales. }\end{array}$ & $\begin{array}{l}\text { El sistema } 1 \text { se correlaciona con } \\
\text { velocidades rápidas, procesamiento } \\
\text { paralelo y automaticidad, mientras } \\
\text { que el Sistema } 2 \text { normalmente está } \\
\text { correlacionado con velocidades lentas; }\end{array}$ \\
\hline $\begin{array}{l}\text { Elna } \\
\text { Schirrmeister } \\
\text { | Anne-Louise } \\
\text { Göhring | } \\
\text { Philine Warnke }\end{array}$ & $\begin{array}{l}\text { Psychological biases and heuristics in } \\
\text { the context of foresight and scenario } \\
\text { processes./ }\end{array}$ & $\begin{array}{l}\text { Esbozar la relevancia de la heurística } \\
\text { y los sesgos seleccionados en los } \\
\text { procesos de escenario, mediante la } \\
\text { implementación de una visión más } \\
\text { completa y mirando los mecanismos } \\
\text { cognitivos con una doble perspectiva }\end{array}$ & $\begin{array}{l}\text { Los humanos no son criaturas } \\
\text { perfectamente racionales. Se basan en } \\
\text { la heurística o la intuición basada en } \\
\text { procesos asociativos. Esto les permite } \\
\text { decidir de manera eficiente, pero } \\
\text { también los hace propensos a errores } \\
\text { sistemáticos (sesgos). }\end{array}$ \\
\hline $\begin{array}{l}\text { López y Marván } \\
\text { (2004) }\end{array}$ & $\begin{array}{l}\text { Validación de una escala de } \\
\text { afrontamiento frente a riesgos } \\
\text { extremos. Salud Publica de } \\
\text { Mexico, } 46 \text { (3), 216-221. }\end{array}$ & $\begin{array}{l}\text { Validar, en población mexicana, una } \\
\text { escala de afrontamiento, adaptada de la } \\
\text { escala francesa "Echèlle Toulousaine de } \\
\text { Coping }\end{array}$ & $\begin{array}{l}\text { La escala de afrontamiento que } \\
\text { proponemos es confiable y válida para la } \\
\text { población mexicana }\end{array}$ \\
\hline
\end{tabular}




\begin{tabular}{|c|c|c|c|}
\hline Autor(es) & Título/Año & Objetivo & Conclusiones \\
\hline $\begin{array}{l}\text { Moynihan T. } \\
(2000)\end{array}$ & $\begin{array}{l}\text { Coping with 'requirements- } \\
\text { uncertainty': the theories-of-action } \\
\text { of experienced IS/software project } \\
\text { managers. } 2000\end{array}$ & $\begin{array}{l}\text { Identificar las estrategias que } \\
\text { experimentan los gerentes de proyectos } \\
\text { para hacer frente a la incertidumbre de } \\
\text { los requisitos. }\end{array}$ & $\begin{array}{l}\text { A medida que aumenta el nivel de } \\
\text { incertidumbre de los requisitos de los } \\
\text { usuarios, se aconseja a los directores } \\
\text { de proyecto se alejen del modelo } \\
\text { tradicional de ciclo de vida de la cascada } \\
\text { hacia enfoques más "experimentales", } \\
\text { como la entrega incremental y la } \\
\text { creación de prototipos. }\end{array}$ \\
\hline $\begin{array}{l}\text { Thomas R.W., } \\
\text { Fugate B.S., } \\
\text { Koukova N.T. } \\
(2011)\end{array}$ & $\begin{array}{l}\text { Coping with time pressure and } \\
\text { knowledge sharing in buyer-supplier } \\
\text { relationships } 2011\end{array}$ & $\begin{array}{l}\text { Desarrollar la noción de que los TPCM } \\
\text { por sus siglas en inglés (Time Pressure } \\
\text { Coping Mechanisms), afectan el } \\
\text { intercambio de conocimientos en las } \\
\text { relaciones comprador-proveedor. }\end{array}$ & $\begin{array}{l}\text { Cada vez más, se está encomendando } \\
\text { a los proveedores que pasen grandes } \\
\text { cantidades de tiempo con clientes } \\
\text { estratégicos con el fin de promover el } \\
\text { intercambio y la coordinación eficaces } \\
\text { de los conocimientos entre los miembros } \\
\text { de la cadena de suministro. }\end{array}$ \\
\hline
\end{tabular}

Tabla 7. Escala de Afrontamiento ante la percepción de un riesgo (Échelle Toulousaine de Coping)

\begin{tabular}{|c|c|c|c|c|c|}
\hline \multirow{2}{*}{ Situación } & \multicolumn{5}{|c|}{ Escala } \\
\hline & 1 & 2 & 3 & 4 & 5 \\
\hline \multicolumn{6}{|l|}{ Acepto la situación pues es inevitable } \\
\hline \multicolumn{6}{|l|}{ Deseo un milagro y ruego a Dios para que me ayude } \\
\hline \multicolumn{6}{|l|}{ Rechazo la idea de que esta situación es grave } \\
\hline \multicolumn{6}{|l|}{ Algunas veces no hago lo que ya había previsto hacer } \\
\hline \multicolumn{6}{|l|}{ Analizo las circunstancias para saber que hacer } \\
\hline \multicolumn{6}{|l|}{ Bromeo y tomo las cosas a la ligera } \\
\hline \multicolumn{6}{|l|}{ Busco actividades para pensar en otra cosa } \\
\hline \multicolumn{6}{|l|}{ Busco información con personas que saben } \\
\hline \multicolumn{6}{|l|}{ Consulto sobre el problema con profesionales } \\
\hline \multicolumn{6}{|l|}{ Controlo en todo momento mis emociones } \\
\hline \multicolumn{6}{|l|}{ Hablo con mi familia para compartir emociones } \\
\hline \multicolumn{6}{|l|}{ Hago como si el peligro no existiera } \\
\hline \multicolumn{6}{|l|}{ Hago frente directamente a la situación } \\
\hline \multicolumn{6}{|l|}{ Hago modificaciones en mi entorno para evitar un desastre } \\
\hline \multicolumn{6}{|l|}{ He establecido mi propio plan de prevención y lo pongo en marcha } \\
\hline \multicolumn{6}{|l|}{ Me es difícil describir lo que siento frente a esta situación } \\
\hline \multicolumn{6}{|l|}{ Me fijo objetivos y redoblo esfuerzos } \\
\hline \multicolumn{6}{|l|}{ Me paseo para distraerme } \\
\hline \multicolumn{6}{|l|}{ Participo más en actividades de protección civil } \\
\hline \multicolumn{6}{|l|}{ Reflexiono sobre las estrategias a utilizar } \\
\hline \multicolumn{6}{|l|}{ Sigo lo que hacen los demás } \\
\hline \multicolumn{6}{|l|}{ Tengo un plan preventivo y lo sigo } \\
\hline \multicolumn{6}{|l|}{ Trato de cambiar mis hábitos de vida en función del problema } \\
\hline \multicolumn{6}{|l|}{ Trato de no pensar en el problema } \\
\hline \multicolumn{6}{|l|}{ Trato de no precipitarme y de reflexionar sobre los pasos a seguir } \\
\hline Trato de no sentir nada & & & & & \\
\hline
\end{tabular}

Fuente: López y Marván (2004)

Notas: 1.Nunca, 2.Pocas veces, 3.Algunas veces, 4.La mayoría de las veces, 5.- Siempre. 


\section{CONCLUSIONES}

La acuacultura es una actividad que genera desarrollo regional en los enclaves donde se implementa. Aun y cuando la pandemia del Covid-19 ha afecta su desempeño, las UPC, siguen produciendo bajo condiciones de riesgos internos y externos. Los administradores, productores y técnicos, no sólo tienen que manejar la disminución de densidades de siembra, sanitización extensiva y medidas de prevención, aplicación de probióticos y prebióticos, para paliar la incidencia de enfermedades y epizootias. Sino que además diseñan su política de costos para afrontar la caída de precios. En la toma de decisiones de los administradores de las UPC, se conjugan tanto elementos de la teoría tradicional de la administración de empresas, y de la Teoría Prospectiva que indican que su operación, se ha manejado tanto desde el conocimiento técnico especializado, como desde un empirismo no estrictamente racional, dado que estas UPC son administradas por individuos que buscan medios activos o pasivos para afrontar las dificultades, con sus respectivos sesgos cognitivos.

De acuerdo con la revisión teórica, entre las características de los heurísticos que generan respuestas inmediatas, no sólo para cuestiones de gran complejidad, sino también para aspectos de probabilidad, frecuencia y predicción. Así pues, se puede establecer por semejanza que los administradores de las UPC utilizan métodos heurísticos, y lo hacen de manera consuetudinaria a través de la experiencia de sus análogos, cuando implementan la toma de la mayoría de las decisiones, que luego derivan en las formas de afrontamiento. Finalmente, mediante la búsqueda bibliográfica, se ha encontrado un instrumento que mide dicho afrontamiento.

\section{AGRADECIMIENTOS}

Se agradece al Centro de Investigación en Alimentación y Desarrollo AC y al CONACYT por el otorgamiento de la beca de postgrado.

\section{BIBLIOGRAFIA}

Ariely, D. 2009. Predictably irrational, revised and expanded edition, Harper Collings Publishers, London.

Bartolini Rosales J. L. y Torres García P. 2004. La camaronicultura en México. Revista Ciencias UNAM. No. 76. Octubre-diciembre. 2004.

Higuera I. y Chavez M. 2013. Manual de Buenas Prácticas de Producción Acuícola de Camarón para la Inocuidad Alimentaria, CIAD AC, SENASICA. ISBN: 968-5384-045
CONAPESCA 2017. Anuario Estadístico 2017. México: SAGARPA.

Bastidas Plaza Héctor Eduardo. 2006. El fideicomiso como mecanismo de garantía y medio de captación de recursos para el procesamiento y comercialización del camarón". Tesis Licenciatura, Facultad de Ciencias Humanísticas y Económicas. Escuela Superior Politécnica Del Litoral. Guayaquil, Ecuador. 220 p.

FAO 2005. DOCUMENTO TÉCNICO DE PESCA 476 Introducciones y movimiento de dos especies de camarones peneidos en Asia y el Pacífico

FAO 2019. GLOBEFISH - Información y Análisis sobre el Comercio Mundial de Pescado. Recuperado de: http://www.fao.org/in-action/globefish/marketreports/ resource-detail/es/c/1241384/.

FIRA 2020. Dirección de Investigación y evaluación Económica y Sectorial. Perspectivas.

Gigerenzer, G. 2011. Heuristic Decision Making, The Annual Review of Psychology, online at psych.annualreviews. org.

Gómez-Gil B., Roque A., y Guerra-Flores A.L. 2001. Enfermedades Infecciosas más comunes en la camaronicultura en México y el impacto del uso de antimicrobianos. México. En: Páez-Osuna F (ed) camaronicultura y medio ambiente.ICMyL-UNAM.

Kahneman, D y Tversky, A. 1979. Prospect Theory: An Analysis of Decision Under Risk, Ecomometrica, Vol. 47, Marzo; No. 2 Pags. 263-291.

Kahneman, D. 2012. Pensar rápido, pensar despacio books. google.com

Lagarda-Leyva, Ernesto A. 2016. Evaluación del desempeño de la cadena de suministro del camarón blanco de agua dulce. Revista Internacional Administración \& Finanzas, v. 9 (1) p. 33-55.

Lawson, M. A. Larrick, R.P., Soll, J.B. 2020. Comparing fast thinking and slow thinking: The relative benefits of interventions, individual differences, and inferential rules./ Judgment and Decision Making, Vol. 15, No. 5, September 2020, pp. 660-684

Lipshitz, R. y Strauss, O. (1997). Coping with Uncertainty: A Naturalistic Decision-Making Analysis Organizational behavior and human decision processes. Vol. 69, No. 2, February, pp. 149-163, Article No. OB972679.

Lopez-Tellez, Norma A. 2020. History, Impact, and Status of Infectious Diseases of the Pacific White Shrimp Penaeus vannamei (Bonne, 1831) Cultivated in Mexico. Journal of the World Aquaculture Society. Volume 51, Issue 2, Page 334.

López-Vázquez E, Marván ML. 2004. Validación de una escala de afrontamiento frente a riesgos extremos. Salud Publica Mex 2004;46:216-22

Mullainathan, S. y Thaler, R. 2000. Behavioral Economics, National Bureau of Economic Research, working paper 7948. 
Muñoz Palma, M., Avilés Ochoa, E., Miranda Espinoza, E.L., 2016. La Lógica Difusa para la evaluación económica y financiera de opciones cambiarias: El caso de la producción acuícola. / International Journal of Information Systems and Software Engineering for Big Companies.

Moynihan T. , 2000. Coping with 'requirementsuncertainty': the theories-of-action of experienced IS/ software project managers. https://doi.org/10.1016/ S0164-1212(00)00049-2

Organización de las Naciones Unidas para la Alimentación y la Agricultura (FA0, 2016). Visión general del sector acuícola nacional, México. http://www.fao.org/fishery/ countrysector/naso_mexico/es
Schirrmeister, E., Göhring A-L., Warnke, P. 2016. Psychological biases and heuristics in the context of foresight and scenario processes.

SIACON, 2019. Sistema de Información Agroalimentaria de Consulta. https://www.gob.mx/siap/documentos/siaconng-161430

Stoner, J. y Freeman, E. 2000. Administración. México. Editorial Prentice-Hall.

USTIC, 2020. United States International Trade Comission. Recuperado de https://www.usitc.gov/ 